\title{
CONTINUUM SHAPE SENSITIVITY FOR NONLINEAR AEROELASTIC GUST RESPONSE
}

\section{Robert Canfield}

VIRGINIA POLYTECHNIC INST AND STATE UNIVERSITY

09/24/2014

Final Report 


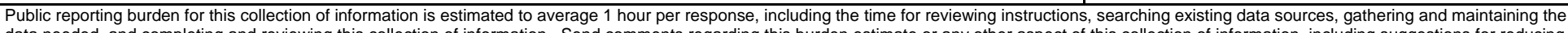

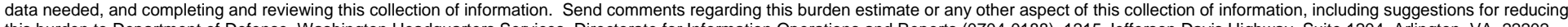

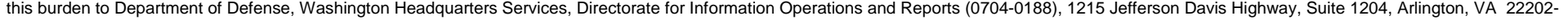

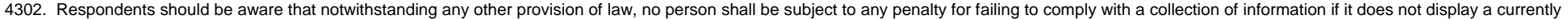
valid OMB control number. PLEASE DO NOT RETURN YOUR FORM TO THE ABOVE ADDRESS.

\begin{tabular}{l|l|l} 
1. REPORT DATE (DD-MM-YYYY) & 2. REPORT TYPE & 3. DATES COVERED (FrOm - To)
\end{tabular}

14-09-2014 $\quad$ Final

\section{TITLE AND SUBTITLE}

June 2011-June 2014

Continuum Shape Sensitivity For Nonlinear Aeroelastic Gust Response

5a. CONTRACT NUMBER

\section{5b. GRANT NUMBER}

FA9550-11-1-0117

5c. PROGRAM ELEMENT NUMBER

$61102 \mathrm{~F}$

6. AUTHOR(S)

Canfield, Robert A.

5d. PROJECT NUMBER

5e. TASK NUMBER

5f. WORK UNIT NUMBER

7. PERFORMING ORGANIZATION NAME(S) AND ADDRESS(ES)

Virginia Polytechnic Institute \& State University (Virginia Tech)

1880 Pratt Dr, Ste 2006

8. PERFORMING ORGANIZATION REPORT NUMBER

Blacksburg, VA 24060-6750

450096

9. SPONSORING / MONITORING AGENCY NAME(S) AND ADDRESS(ES)

10. SPONSOR/MONITOR'S ACRONYM(S)

AFOSR

USAF, AFRL, AF Office of Scientific Research

875 N. Randolph St., Room 3112

Arlington, VA 22203

11. SPONSOR/MONITOR'S REPORT NUMBER(S)

\section{DISTRIBUTION / AVAILABILITY STATEMENT}

Distribution A - Approved for Public Release

\section{SUPPLEMENTARY NOTES}

\section{ABSTRACT}

The objective of this research was to derive and apply new computational algorithms for the aeroelastic design sensitivity of high fidelity, coupled fluid-structure interaction (FSI) problems. A continuum sensitivity analysis (CSA) method was developed for efficient and accurate computation of shape design derivatives for nonlinear aeroelastic structures. The local and total forms for CSA were compared for the first time, based on their accuracy, efficiency and implementation. A proof was published for equivalence of the total-form CSA method and the discrete analytic method-for the first time for general nonlinear transient second-order partial differential equations that govern many field problems. Accuracy of the local-form CSA was increased in two ways. One was to use higher-order p-elements. The other way was to use spatial gradient reconstruction (SGR) of the analysis output to compute the sensitivity boundary conditions. SGR for the structural domain was improved by reconstructing gradients of the Neumann sensitivity boundary conditions directly from force and moment output of the structural analysis instead of reconstructing higher-order derivatives. Guidelines were developed for defining SGR patches with controllable accuracy and rate of convergence for CSA.

\section{SUBJECT TERMS}

gradient-based optimization, continuous sensitivity, continuum sensitivity, variational sensitivity, design derivatives, aeroelasticity

\begin{tabular}{|l|l|l|l|l|l|}
\hline \multicolumn{2}{|l|}{ 16. SECURITY CLASSIFICATION OF: } & $\begin{array}{l}\text { 17. LIMITATION } \\
\text { OF ABSTRACT }\end{array}$ & $\begin{array}{l}\text { 18. NUMBER } \\
\text { OF PAGES }\end{array}$ & $\begin{array}{l}\text { 19a. NAME OF RESPONSIBLE PERSON } \\
\text { Robert A. Canfield }\end{array}$ \\
\cline { 1 - 2 } $\begin{array}{l}\text { a. REPORT } \\
\text { U }\end{array}$ & b. ABSTRACT & c. THIS PAGE & UU & 6 & $\begin{array}{l}\text { 19b. TELEPHONE NUMBER (include area } \\
\text { code) } \\
540-231-5981\end{array}$ \\
\end{tabular}




\title{
FINAL PERFORMANCE REPORT
}

14 September 2014

for

CONTINUUM SHAPE SENSITIVITY FOR NONLINEAR AEROELASTIC GUST RESPONSE

\author{
AFOSR GRANT \#FA9550-11-1-0117 \\ Performance period: 15 June 2011 to 14 June 2014 \\ Robert A. Canfield \\ Aerospace and Ocean Engineering Department \\ Virginia Polytechnic Institute and State University
}

AFOSR Program Manager

Dr. Fariba Fahroo 


\title{
CONTINUUM SHAPE SENSITIVITY FOR NONLINEAR AEROELASTIC GUST RESPONSE
}

\author{
Robert A. Canfield
}

\begin{abstract}
The objective of this research was to derive and apply new computational algorithms for the aeroelastic design sensitivity of high fidelity, coupled fluid-structure interaction (FSI) problems. A continuum sensitivity analysis (CSA) method was developed for efficient and accurate computation of shape design derivatives for nonlinear aeroelastic structures. The local and total forms for CSA were compared for the first time, based on their accuracy, efficiency and implementation. A proof was published for equivalence of the total-form CSA method and the discrete analytic method - for the first time for general nonlinear transient second-order partial differential equations that govern many field problems. Accuracy of the local-form CSA was increased in two ways. One was to use higher-order p-elements. The other way was to use spatial gradient reconstruction (SGR) of the analysis output to compute the sensitivity boundary conditions. SGR for the structural domain was improved by reconstructing gradients of the Neumann sensitivity boundary conditions directly from force and moment output of the structural analysis instead of reconstructing higher-order derivatives. Guidelines were developed for defining SGR patches with controllable accuracy and rate of convergence for CSA.
\end{abstract}

\section{Summary}

AFOSR awarded the grant in June 2014. This grant builds upon research begun in AFOSR grant begun in FY11. The three-year follow-on research effort has further developed the computational algorithms for efficient analysis and design optimization of aeroelastic models in several areas. Domain ad boundary velocity approaches, also known as total and local derivative formulations, were compared thoroughly. The remedy for handling interface discontinuities in built-up structures for the boundary velocity CSA approach was extended from beams to unstiffened and stiffened plates. Spatial gradient reconstruction (SGR) was demonstrated as a means to improve CSA accuracy in a controllable and nonintrusive manner. Three journal articles [1-3] were published and four others [4-7] submitted for publication documenting the funded research. Seven conference papers were presented during the performance period [8-14]. Two dissertations were published: http://hdl.handle.net/10919/23282 and http://hdl.handle.net/10919/48589.

Progress was made in the first year in developing continuous sensitivity equations (CSE) for fluid-structure interaction (FSI). The CSE and their associated sensitivity boundary conditions were studied for a built-up joined beam structure under transient aerodynamic loads. For built-up structures, the state variable field and the local sensitivity variable field have different continuity requirements - the former is $\mathrm{C}^{0}$ continuous, while the latter is piecewise $\mathrm{C}^{0}$ continuous. For the discrete system, this might imply a choice of different degrees of freedom, which in turn would alter the assembly procedure for the system matrices. A remedy was provided so that for nonlinear problems, when the Newton-Raphson method is used, the tangent stiffness matrix may still be used as the sensitivity coefficient matrix for solving the linear sensitivity equations with the Galerkin finite element method. The domain velocity (total derivative) formulation of CSE, 
most often used for structural problems in part to avoid local sensitivity discontinuity due to strain discontinuities, and the boundary velocity (local derivative) formulation, most often used for fluid problems, were compared. The discretized domain velocity formulation of the CSE method was shown to be equivalent to the discrete analytical sensitivity formulation for potential flow problems for the first time. As a result, the boundary velocity formulation for CSE is being pursued for FSI problems. Benchmark examples with analytical and finite difference sensitivity results were solved with CSE for the purpose of verification. The boundary velocity CSE formulation was implemented and solved in a "black box" solver without access to the source code in order to numerically solve the sensitivity systems of a nonlinear FSI problem involving determinate and indeterminate (built-up) structures. Sources of inaccuracy in the boundary velocity formulation relative to the domain velocity formulation were investigated.

In the second year progress was made in developing a continuum sensitivity method for efficient and accurate computation of shape design derivatives for nonlinear aeroelastic structures subject to transient aerodynamic loads. The CSEs are often posed in terms of local derivatives (local form) for fluid applications and in terms of total derivatives (total form) for structural applications. The local form and the total forms for static and transient aeroelastic (fluidstructure interaction) problems were compared for the first time, based on their accuracy, efficiency and implementation. The total form CSE exhibited higher accuracy than local form, when the same numerical method was used for solving fluid and structures benchmark problems. The local form requires design velocity only at the boundaries, and the continuity requirements for the design velocity is lower than the total form CSE method. On the other hand, local form requires higher order spatial derivatives to specify its sensitivity boundary conditions than does total form CSE. Accuracy of the local form CSE was increased in two ways. One was to use higher-order $p$-elements. The other way was to use spatial gradient reconstruction of the analysis output to compute the sensitivity boundary conditions. The latter was improved for the structural domain by reconstructing gradients of the Neumann sensitivity boundary conditions directly from force and moment output of the structural analysis instead of reconstructing higher-order derivatives of displacement. The total form continuum sensitivity method was applied for the first time to nonlinear transient gust response. The equivalence of the discrete analytic method and the total form CSE method was proved for the first time for general nonlinear transient second-order partial differential equations that govern many field problems. The equivalence of these two methods was demonstrated for general transient geometric nonlinear elasticity and potential flow, as specific examples.

In the third year, continuum sensitivity analysis (CSA) was matured for efficient and accurate computation of shape design derivatives for nonlinear aeroelastic structures subject to transient aerodynamic loads. An advantage of the local form is that it requires design velocity only at the boundaries, and the continuity requirements for the design velocity is lower than the total-form CSA method. On the other hand, local form requires higher order spatial derivatives to specify its sensitivity boundary conditions than does total-form CSA. Accuracy improvement of the local-form CSA was increased by way spatial gradient reconstruction (SGR) of the analysis output to compute the sensitivity boundary conditions was published for structures for the first time. An advance compared to the previous application for fluids, SGR for the structural domain was improved by reconstructing gradients of the Neumann sensitivity boundary conditions directly from force and moment output of the structural analysis instead of reconstructing higherorder derivatives of displacement. Studies this year indicated that CSA with SGR achieves the 
same rate of convergence as the original analysis, provided sufficient layers are employed in the patches for Taylor series reconstruction. An example demonstrated that local-form CSA with SGR can approach the accuracy achieved using analytical spatial derivatives and can exceed that accuracy of discrete sensitivity or equivalently total-form CSA. Guidelines were developed for defining SGR patches with controllable accuracy and rate of convergence.

\section{Accomplishments}

Several significant contributions to the state of knowledge were made for the application of CSA. Although they were posed in support of the sensitivity analysis of FSI problems, some findings are more general and more fundamental than the application to FSI.

The total form continuum sensitivity method was applied for the first time to nonlinear transient aeroelastic problems. For nonlinear problems, it was shown that the tangent stiffness matrix yields the desired sensitivity coefficient matrix for solving the sensitivity equations. The local form CSE was applied for the first time to built-up structures. The difficulty in implementation was pointed out and resolved [1]. The first comparison of the local form and the total form CSE method for static and transient aeroelastic problems has been made on their accuracy, efficiency and implementation [6]. The equivalence of the discrete analytic method and the total form CSE method is proved for the first time for general nonlinear transient second-order partial differential equations that govern many field problems [2].

The total form CSE method, once discretized, was proven to be equivalent to the discrete analytic method for nonlinear second-order field problems under certain conditions. The conditions are that the two methods use the same: (1) numerical approximation of the field variables and sensitivity variables, (2) integration of the finite element matrices, (3) design velocity fields that are linear with respect to the design variable, and (4) shape functions for domain transformation and for design velocity field calculation. Although, under these conditions, the discrete analytic method is identical to the total form CSE method, the total form CSE can be implemented without satisfying all these conditions. The advantage of the CSE method is that the discrete analytic method requires the knowledge of the implementation details of the original analysis codes, which may be inaccessible or very complex, while the continuum method requires minimum information of the source codes. The continuum method only requires knowledge of the governing equations, the numerical solutions and the spatial gradients of the solutions for the governing equations. Thus, the CSE method can be implemented by treating the existing analysis solver as a "black box" [3].

A primary objective of this research was to develop design sensitivity analysis (DSA) that can accurately and efficiently calculate design derivatives of high fidelity models, such as nonlinear transient aeroelastic gust analysis. Furthermore, the DSA method should be nonintrusive, providing the ability to calculate design derivatives of analyses conducted using general-purpose codes. This specific DSA formulation developed here is called local CSA with spatial gradient reconstruction (SGR). Moreover, an added benefit of using local CSA is that mesh sensitivities are not required. Specific contributions follow.

- This research marks the first application of local CSA for linear [3] and nonlinear analysis [4] of structural models using plate elements. It is the first demonstration of implementing local 
CSA with black box analysis tools without knowledge of finite element shape functions. Furthermore, it is the first application of local CSA to a built-up structure that uses mixed element types (plate and beam elements). Interface boundary conditions were derived for joined mixed elements.

- Local CSA with SGR can be implemented with general purpose codes in a nonintrusive fashion, treating the analysis tool as a "black box." The only requirements are for the user to differentiate the boundary conditions to define sensitivity loads and for the analysis tool to provide as output the system response of primary and secondary variables, as well as the system matrices. The method is element agnostic and can be readily implemented for a variety of analysis models with varying degrees of fidelity. Numerous examples, conducted with Nastran analyses, demonstrated these attributes. The results also demonstrate that the method can be as accurate as other analytic DSA methods and more accurate than most [5].

- SGR has been utilized in the context of flow sensitivities, but this is the first use of SGR in the context of structural sensitivities. Previously, SGR was utilized to approximate high-order derivatives of primary variables to formulate local CSE boundary conditions. The local CSA method with SGR presented here requires only the approximation of first-order derivatives, but of both primary and secondary variables. This is a key ingredient in making the method element-agnostic and amenable to general purpose codes. This research marks the first time that SGR has been employed in this way and for this purpose [3].

- Guidelines were developed to control convergence of accuracy for local CSA with spatial gradient reconstruction. In general, the convergence rate is largely dependent on the accuracy of the spatial derivative approximations that appear in the local CSE boundary conditions. The accuracy of these approximations is driven by the parameters used for the SGR [5].

- A hybrid adjoint was derived to efficiently compute static shape derivatives for many design variables, using a discrete adjoint with CSA for which accuracy is controlled by SGR [7, 14].

\section{Acknowledgment/Disclaimer}

This work was sponsored by the Air Force Office of Scientific Research, USAF, under grant/contract number FA9550-11-1-0117. The views and conclusions contained herein are those of the authors and should not be interpreted as necessarily representing the official policies or endorsements, either expressed or implied, of the Air Force Office of Scientific Research or the U.S. Government.

\section{References}

[1] Liu, Shaobin; Canfield, Robert. A. (2013), "Boundary Velocity Method for Continuum Shape Sensitivity of Nonlinear Fluid-Structure Interaction Problems," Journal of Fluids and Structures, http://dx.doi.org/10.1016/j.jfluidstructs.2013.05.003, June 6, 2013, Vol. 40, pp. 284-301

[2] Liu, Shaobin; and Canfield, Robert. A. (2013), "Equivalence of Continuum and Discrete Analytic Sensitivity Methods for Nonlinear Differential Equations," Structural and Multidisciplinary Optimization, doi: 10.1007/s00158-013-0951-4 (online 4 July 2013), December 2013, Volume 48, Issue 6, pp. 1173-1188 
[3] Cross, David M. and Canfield, Robert A. (2014), "Local continuum shape sensitivity with spatial gradient reconstruction," Structural and Multidisciplinary Optimization, June, 2014, DOI 10.1007/s00158-014-1092-0

[4] Cross, David M.; and Canfield, Robert A., "Local Continuum Shape Sensitivity with Spatial Gradient Reconstruction for Nonlinear Analysis," accepted for publication in Structural and Multidisciplinary Optimization

[5] Cross, David M.; and Canfield, Robert A., "Convergence of Accuracy for Local Continuum Sensitivity Method Using Spatial Gradient Reconstruction," submitted to AIAA Journal

[6] Liu, Shaobin; and Canfield, Robert A., "Two Forms of Continuum Shape Sensitivity Method for Fluid-Structure Interaction Problems," submitted to AIAA Journal

[7] Kulkarni, Mandar D.; Cross, David M.; and Canfield, Robert A., "Discrete Adjoint Formulation for Continuum Sensitivity Analysis," submitted to AIAA Journal

[8] Liu, Shaobin; and Canfield, Robert A., "Continuum Shape Sensitivity Method for Fluid Flow Around an Airfoil," 53rd AIAA/ASME/ASCE/AHS/ASC Structures, Structural Dynamics and Materials Conference, AIAA-2012-1426, Honolulu, Hawaii, Apr. 23-26, 2012

[9] Cross, David; and Canfield, Robert A., "Solving Continuum Shape Sensitivity with Existing Tools for Nonlinear Aeroelastic Gust Analysis," 53rd AIAA/ASME/ASCE/AHS/ASC Structures, Structural Dynamics and Materials Conference, AIAA-2012-1923, Honolulu, Hawaii, Apr. 23-26, 2012

[10] Liu, Shaobin; and Canfield, Robert A., "Continuum Sensitivity Method for Aeroelastic Shape Design Problems," AIAA-2012-5480, 14th AIAA/ISSMO Multidisciplinary Analysis and Optimization Conference, September 17-19, 2012

[11] Cross, David; and Robert Canfield, "Continuum Shape Sensitivity with Spatial Gradient Reconstruction of Nonlinear Aeroelastic Gust Response," AIAA-2012-5597, 14th

AIAA/ISSMO Multidisciplinary Analysis and Optimization Conference. September 17-19, 2012

[12] Cross, David M.; and Canfield, Robert A., "Continuum Shape Sensitivity with Spatial Gradient Reconstruction of Built-up Structures,” DOI: 10.2514/6.2013-1933, 54th AIAA/ASME/ASCE/AHS/ASC Structures, Structural Dynamics, and Materials Conference, April 8-11, 2013

[13] Kulkarni, Mandar D.; Canfield, Robert A.; and Patil, Mayuresh, "Integration of Geometric Sensitivity and Spatial Gradient Reconstruction for Aeroelastic Shape Optimization," AIAA2014-0470, AIAA SciTech 10th Multidisciplinary Design Optimization Conference, National Harbor, Maryland, January 13-17, 2014

[14] Kulkarni, Mandar D.; Canfield, Robert A.; and Patil, Mayuresh, "Nonintrusive Continuum Sensitivity Analysis for Aerodynamic Shape Optimization,” AIAA-2014-2043, 15th AIAA/ISSMO Multidisciplinary Analysis and Optimization Conference, Atlanta, Georgia, June 16, 2014 